\title{
A Domain-independent Rule-based Framework for Event Extraction
}

\author{
Marco A. Valenzuela-Escárcega Gus Hahn-Powell Thomas Hicks Mihai Surdeanu \\ University of Arizona, Tucson, AZ, USA \\ \{marcov, hahnpowell, msurdeanu, hickst\}eemail.arizona.edu
}

\begin{abstract}
We describe the design, development, and API of ODIN (Open Domain INformer), a domainindependent, rule-based event extraction (EE) framework. The proposed EE approach is: simple (most events are captured with simple lexico-syntactic patterns), powerful (the language can capture complex constructs, such as events taking other events as arguments, and regular expressions over syntactic graphs), robust (to recover from syntactic parsing errors, syntactic patterns can be freely mixed with surface, token-based patterns), and fast (the runtime environment processes 110 sentences/second in a real-world domain with a grammar of over 200 rules). We used this framework to develop a grammar for the biochemical domain, which approached human performance. Our EE framework is accompanied by a web-based user interface for the rapid development of event grammars and visualization of matches. The ODIN framework and the domain-specific grammars are available as open-source code.
\end{abstract}

\section{Introduction}

Rule-based information extraction (IE) has long enjoyed wide adoption throughout industry, though it has remained largely ignored in academia, in favor of machine learning (ML) methods (Chiticariu et al., 2013). However, rule-based systems have several advantages over pure ML systems, including: (a) the rules are interpretable and thus suitable for rapid development and domain transfer; and (b) humans and machines can contribute to the same model. Why then have such systems failed to hold the attention of the academic community? One argument raised by Chiticariu et al. is that, despite notable efforts (Appelt and Onyshkevych, 1998; Levy and Andrew, 2006; Hunter et al., 2008; Cunningham et al., 2011; Chang and Manning, 2014), there is not a standard language for this task, or a "standard way to express rules", which raises the entry cost for new rule-based systems.

Here we aim to address this issue with a novel event extraction (EE) language and framework called ODIN (Open Domain INformer). We follow the simplicity principles promoted by other natural language processing toolkits, such as Stanford's CoreNLP, which aim to "avoid over-design", "do one thing well", and have a user "up and running in ten minutes or less" (Manning et al., 2014). In particular, our approach is:

Simple: Taking advantage of a syntactic dependency ${ }^{1}$ representation (de Marneffe and Manning, 2008), our EE language has a simple, declarative syntax (see Examples $1 \& 2$ ) for $n$-ary events, which captures single or multi-word event predicates (trigger) with lexical and morphological constraints, and event arguments (e.g., theme) with (generally) simple syntactic patterns and semantic constraints.

Powerful: Despite its simplicity, our EE framework can capture complex constructs when necessary, such as: (a) recursive events ${ }^{2}$, (b) complex regular expressions over syntactic patterns for event arguments. Inspired by Stanford's Semgrex ${ }^{3}$, we have extended a standard regular expression language to describe patterns over directed graphs ${ }^{4}$, e.g., we introduce new $<$ and $>$ operators to specify the direction of edge traversal in the dependency graph. Finally, we allow for (c) optional arguments ${ }^{5}$ and multiple arguments with the same name.

Robust: To recover from unavoidable syntactic errors, SD patterns (such as the ones in Examples 1 and 2) can be can be freely mixed with surface, token-based patterns, using a language inspired by the Allen Insti-

\footnotetext{
${ }^{1}$ Hereafter abbreviated as SD.

${ }^{2}$ Events that take other events as arguments (see Figure 1 and the corresponding Example (2) for such an event in the biochemical domain. The Positive_Regulation takes a Phosphorylation event as the Controlled argument)

${ }^{3}$ nlp. stanford.edu/software/tregex. shtml

${ }^{4}$ Here we use syntactic dependencies.

${ }^{5}$ cause in Example 1.
} 
TopBP1 promotes the phosphorylation of cyclin-D1 by ATR.

Phosphorylation ( Theme=cyclin-D1, Cause=ATR)

Figure 1: An example sentence containing a recursive event.

tute of Artificial Intelligence's Tagger ${ }^{6}$. These patterns match against information extracted in our text processing pipeline ${ }^{7}$, namely a token's part of speech, lemmatized form, named entity label, and the immediate incoming and outgoing edges in the SD graph. Example 3 shows an equivalent rule to the one in Example 1 using surface patterns (i.e. a pattern that is independent of a token sequence's underlying syntactic structure).

Fast: Our EE runtime is fast because our rules use event trigger phrases, captured with shallow lexicomorphological patterns, as starting points. Only when event triggers are detected is the matching of more complex syntactic patterns for arguments attempted. This guarantees quick executions. For example, in the biochemical domain (discussed in Section 2), our framework processes an average of 110 sentences/second $^{8}$ with a grammar of 211 rules on a laptop with an i7 CPU and 16GB of RAM.

\section{Building a Domain from Scratch}

We next describe how to use the proposed framework to build an event extractor for the biochemical domain (Ohta et al., 2013) from scratch.

Rule-based systems have been shown to perform at the state-of-the-art for event extraction in the biology domain (Peng et al., 2014; Bui et al., 2013). The domain, however, is not without its challenges. For example, it is not uncommon for biochemical events to contain other events as arguments. Consider the example sentence in Figure 1. The sentence contains two events, one event referring to the biochemical process known as phosphorylation, and a recursive event describing a biochemical regulation that controls the mentioned phosphorylation. We will introduce a minimal set of rules that capture these two events. Here, we will assume the simple entities (denoted in bold in Figure 1) have already been detected through a named entity recognizer. ${ }^{9}$

When a rule matches, the extracted token spans for trigger and arguments, together with the corresponding event and argument labels (here the event

\footnotetext{
${ }^{6}$ https://github.com/allenai/taggers

${ }^{7}$ https://github.com/sistanlp/ processors

${ }^{8}$ after the initial text processing pipeline

${ }^{9}$ Though the discussion focuses on event extraction, our framework can also be applied to the task of entity recognition.
}

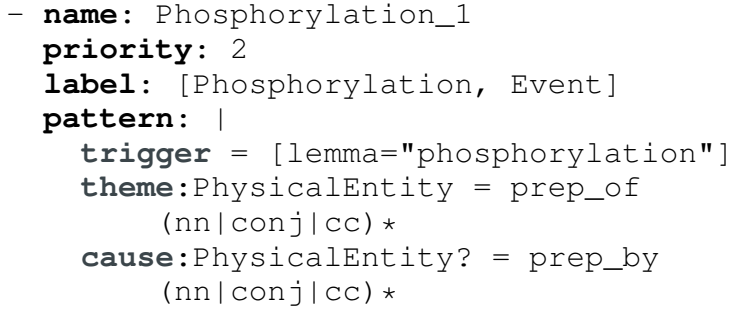

Example 1: An example of a rule using syntactic structure. For the phosphorylation event, our selected event trigger (LINE 5) is a nominal predicate with the lemma phosphorylation. This trigger serves as the starting point for the syntactic patterns that extract event arguments. When searching for a theme to the Phosphorylation event, we begin at the specified trigger and look for an incoming dependent that is the object of the preposition of. The pattern fragment $(\mathrm{nn} \mid \mathrm{conj}$ jand $\mid \mathrm{CC})$ * targets entities that appear as modifiers in noun phrases (e.g., ... of the cyclin-D1 protein), or a series of arguments in a coordinated phrase. The entity mention associated with our theme must be a named entity with the label PhysicalEntity (LINE 7), a hypernym of several more specialized types identified in an earlier iteration. The cause argument is marked as optional (denoted by the ? symbol).

label is Phosphorylation, and the argument labels are theme \& cause) are dispatched to a labeling action. By default, these actions simply create an EventMention Scala object with the corresponding event label, and the extracted named arguments. Example 5 summarizes the EventMention class. Custom actions may be defined as Scala code, and be attached to specific rules. For example, a custom action may trigger coreference resolution when a rule matches a common noun, e.g., the protein, instead of the expected named entity.

The second rule, shown in Example 2, captures the recursive event in Figure 1. Importantly, this rule takes other events as arguments, e.g., the controlled argument must be an event mention, here generated by the rule in Example 1. To guarantee correct execution, the runtime repeatedly applies the given EE grammar on each sentence until no rule matches. For example, here the rule in Example 2 would not match in the first 


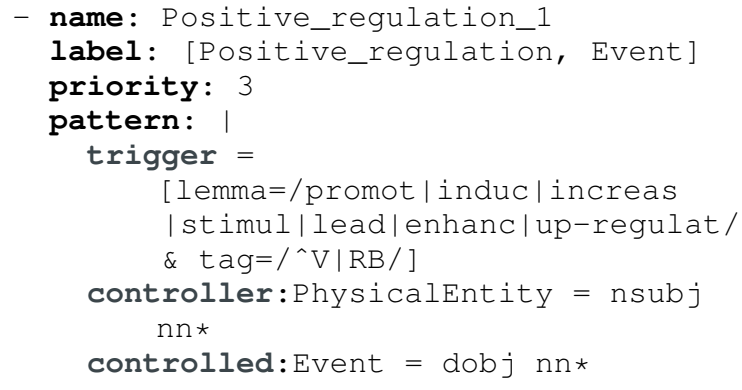

Example 2: An example of a rule designed to capture a recursive event. The rule detects a relevant verbal or adverbial trigger and expects its arguments to be in a SUBJECT $\leftrightarrow$ DIRECT OBJECT relationship. The controlled argument must be the mention of another event.

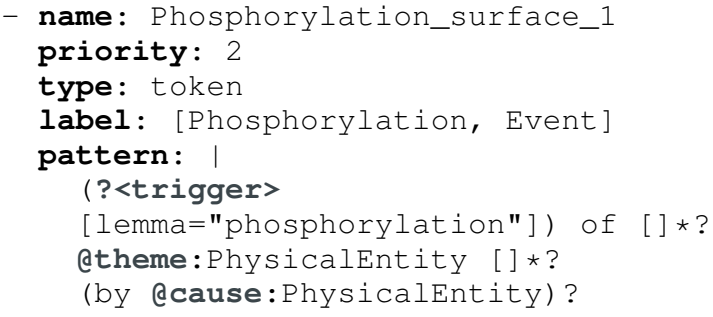

Example 3: An alternative rule to Example 1 that uses a surface pattern. Surface patterns match event triggers and arguments over sequences of tokens and other mentions (e.g., the theme matches over an entire named entity of type PhysicalEntity). Event triggers (trigger) match the whole sequence of tokens encompassed in parentheses. Argument names preceded by the a symbol, e.g., atheme, require the specification of an event type (denoted by :type). This pattern is shorthand for matching the span of an entire named entity with the specified type.

iteration because no event mentions have been created yet, but would match in the second iteration. This process can optionally be optimized with rule priorities (as shown in the figure). For example, the priorities assigned to Examples 1 and 2 enforce that the second rule is executed only in an iteration following the first rule. Utilizing rule priorities allows for a derivational construction of complex events or complete grammars from their components.

Once the grammar has been defined, the entire system can be run in less than 10 lines of code, as shown in Example 4. The output of this code is a collection of event mentions, i.e., instances of the EventMention class outlined in Example 5.

\section{Visualization}

We accompany the above EE system with an interactive web-based tool for event grammar development and re-

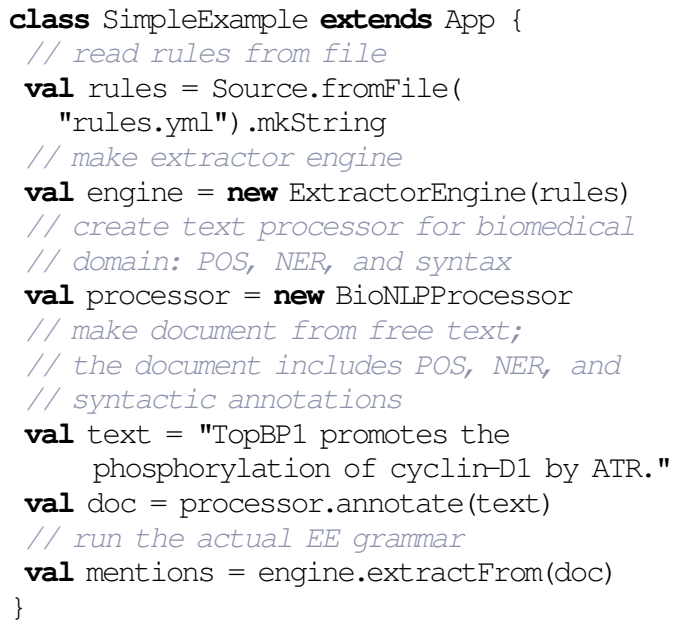

Example 4: The minimal Scala code required to run the system. The input (LINE 13) is raw text. The output is a list of event mentions of the type EventMention. Here we show the use of a text processor specific to the biomedical domain. The framework also includes an opendomain text processor that includes POS tagging, named entity recognition, syntactic parsing, and coreference resolution. Additional processors for domain-specific tasks can easily be added.

sults visualization. Figure 2 shows the input fields for the user interface. The UI accepts free text to match against, and can be configured to run either a predefined domain grammar or one provided on-the-fly through a text box, allowing for the rapid development and tuning of rules.

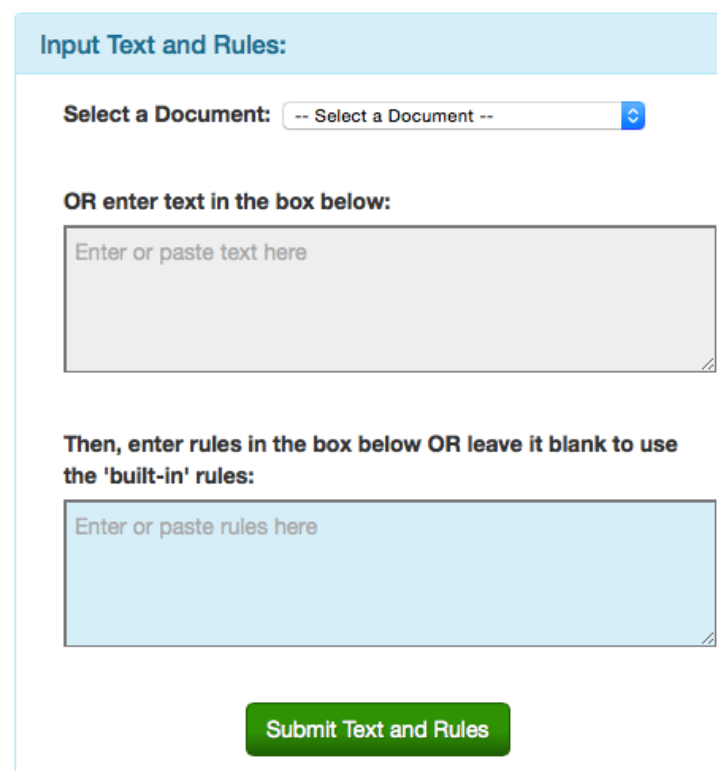

Figure 2: Our interactive environment for rapid development of event grammars. The UI supports the input of rules and free text.

Figure 3 shows the output of the visualization tool on the example sentence from Figure 1 using the gram- 


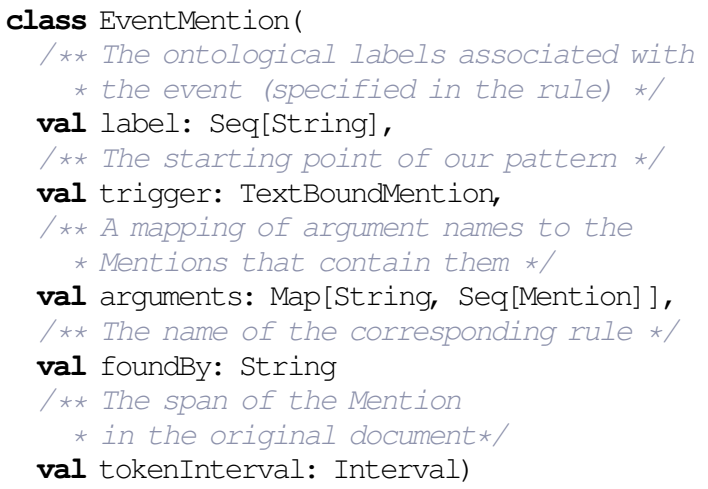

Example 5: Example 4 produces a set of mentions. Here we focus on mentions of events (EventMention). This code block shows relevant fields in the EventMention class, which stores each event mention detected and assembled by the system. The arguments field captures the fact that the mapping from names to arguments is one-to-many (e.g., there may be multiple theme arguments). Interval stores a token span in the input text. TextBoundMention stores a simple mention, minimally a label and a token span.

mar discussed in the previous section. The web interface is implemented as a client-server Grails ${ }^{10}$ web application which runs the EE system on the server and displays the results on the client side. The application's client-side code displays both entity and event mentions, as well as the output of the text preprocessor (to help with debugging) using Brat (Stenetorp et al., 2012).

\section{Results}

We extended the grammar introduced previously to capture 10 different biochemical events, with an average of 11 rules per event type. Using this grammar we participated in a recent evaluation by DARPA's Big Mechanism program ${ }^{11}$, where systems had to perform deep reading of two research papers on cancer biology. Table 1 summarizes our results.

Our system was ranked above the median, with respect to overall F1 score. We find these results encouraging for two reasons. First, inter-annotator agreement on the task was below $60 \%$, which indicates that our system roughly approaches human performance, especially for precision. Second, the lower recall is partially explained by the fact that annotators marked also indirect biological relations (e.g., $A$ activates $B$ ), which do not correspond to actual biochemical reactions but, instead, summarize sequences of biochemical reactions. Our grammar currently recognizes only direct biochemical reactions.

\footnotetext{
${ }^{10}$ https: //grails.org

${ }^{11}$ http: / / www.darpa.mil/Our_Work/I20/ Programs/Big_Mechanism.aspx
}

\begin{tabular}{r|r|r|r} 
System & Precision & Recall & F1 \\
\hline Submitted run & $54 \%$ & $29 \%$ & $37.3 \%$ \\
\hline Ceiling system & $82.1 \%$ & $81.8 \%$ & $82 \%$
\end{tabular}

Table 1: Results from the January 2015 DARPA Big Mechanism Dry Run evaluation on reading biomedical papers, against a known biochemical model. In addition to event extraction, this evaluation required participants to identify if the extracted information corroborates, contradicts, or extends the given model. Here, extending the model means proposing a biochemical reaction that is not contained in the model, but it involves at least a biochemical entity from the model. The ceiling system indicates idealized performance of the rule-based framework, after a post-hoc analysis.

More importantly, this evaluation offers a good platform to analyze the potential of the proposed rule-based framework, by estimating the ceiling performance of our EE system, when all addressable issues are fixed. We performed this analysis after the evaluation deadline, and we manually:

1. Removed the keys that do not encode direct biochemical reactions.

2. Corrected three rules, to better model one event and one entity type.

3. Fixed system bugs, including XML parsing errors, which caused some meta data to appear in text and be misinterpreted as biological entities, and a syntax error in one rule, which caused several false positives.

The results of this ceiling system are listed in the second row in Table 1. This analysis highlights an encouraging finding: the current rule framework is expressive: it can capture approximately $80 \%$ of the events in this complex domain. The remaining $20 \%$ require coreference resolution and complex syntactic patterns, which were not correctly captured by the parser.

\section{Related Work}

Despite the dominant focus on machine learning models for IE in the literature, previous work includes several notable rule-based efforts. For example, GATE (Cunningham et al., 2011), and the Common Pattern Specification Language (Appelt and Onyshkevych, 1998) introduce a rule-based framework for IE, implemented as a cascade of grammars defined using surface patterns. The ICE system offers an active-learning system that learns named entity and binary relation patterns built on top of syntactic dependencies (He and Grishman, 2011). Stanford's Semgrex ${ }^{12}$ and Tregex (Levy and Andrew, 2006) model syntactic patterns,

\footnotetext{
${ }^{12}$ http://nlp.stanford.edu/software/ tregex.shtml
} 


\section{References}

Appelt, Douglas E and Boyan Onyshkevych. 1998. The common pattern specification language. In Proc. of the TIPSTER Workshop. pages 23-30.

Bui, Quoc-Chinh, Erik M Van Mulligen, David Campos, and Jan A Kors. 2013. A fast rule-based approach for biomedical event extraction. Proc. of ACL page 104.

Chang, Angel X. and Christopher D. Manning. 2014. TokensRegex: Defining cascaded regular expressions over tokens. Technical Report CSTR 2014-02, Computer Science, Stanford.

Chiticariu, Laura, R. Krishnamurthy, Y. Li, F. R. Reiss, and S. Vaithyanathan. 2011. Domain adaptation of rule-based annotators for named-entity recognition tasks. In Proc. of EMNLP.

Chiticariu, Laura, Yunyao Li, and Frederick R Reiss. 2013. Rule-based information extraction is dead! long live rule-based information extraction systems! In Proc. of EMNLP.

Cunningham, Hamish, Diana Maynard, Kalina Bontcheva, Valentin Tablan, Niraj Aswani, Ian Roberts, Genevieve Gorrell, Adam Funk, Angus Roberts, Danica Damljanovic, Thomas Heitz, Mark A. Greenwood, Horacio Saggion, Johann Petrak, Yaoyong Li, and Wim Peters. 2011. Developing Language Processing Components with GATE (Version 6). University of Sheffield.

de Marneffe, Marie-Catherine and Christopher D. Manning. 2008. The Stanford typed dependencies representation. In Proc. of COLING Workshop on Cross-framework and Cross-domain Parser Evaluation.

He, Yifan and Ralph Grishman. 2011. Ice: Rapid information extraction customization for nlp novices. In Proc. of NAACL.

Huang, Minlie, Xiaoyan Zhu, Yu Hao, Donald G. Payan, Kunbin Qu, and Ming Li. 2004. Discovering patterns to extract proteinprotein interactions from full texts. Bioinformatics 20(18):3604-3612.

Hunter, Lawrence, Zhiyong Lu, James Firby, William A Baumgartner, Helen L Johnson, Philip V Ogren, and K Bretonnel Cohen. 2008. Opendmap: an open source, ontology-driven concept analysis engine, with applications to capturing knowledge regarding protein transport, protein interactions and cell-type-specific gene expression. BMC bioinformatics $9(1): 78$.

Levy, Roger and Galen Andrew. 2006. Tregex and Tsurgeon: tools for querying and manipulating tree data structures. In Proc. of LREC.

Manning, C. D., M. Surdeanu, J. Bauer, J. Finkel, S. J. Bethard, and D. McClosky. 2014. The Stanford CoreNLP natural language processing toolkit. In Proc. of ACL.

Ohta, Tomoko, Sampo Pyysalo, Rafal Rak, Andrew Rowley, Hong-Woo Chun, Sung-Jae Jung, Sung-Pil Choi, Sophia Ananiadou, and Junichi Tsujii. 2013. Overview of the pathway curation (pc) task of bionlp shared task 2013. In Proc. of the BioNLP-ST Workshop.

Peng, Yifan, Manabu Torii, Cathy $\mathrm{H} \mathrm{Wu}$, and $\mathrm{K}$ VijayShanker. 2014. A generalizable NLP framework for fast development of pattern-based biomedical relation extraction systems. BMC bioinformatics 15(1):285.

Stenetorp, Pontus, Sampo Pyysalo, Goran Topić, Tomoko Ohta, Sophia Ananiadou, and Jun'ichi Tsujii. 2012. Brat: a web-based tool for nlp-assisted text annotation. In Proc. of the Demonstrations at EACL. 